\title{
Publisher Correction: Neurovascular coupling and oxygenation are decreased in hippocampus compared to neocortex because of microvascular differences
}

\author{
K. Shaw (1), L. Bell, K. Boyd, D. M. Grijseels (1D, D. Clarke, O. Bonnar (1), H. S. Crombag (1) \& C. N. Hall(1)
}

Correction to: Nature Communications https://doi.org/10.1038/s41467-021-23508-y, published online 27 May 2021

The original version of this Article contained errors in Eq. (1), in which $\operatorname{Hbt}(\mathrm{t})$ was used as a numerator and denominator instead of $\operatorname{Hbr}(\mathrm{t})$.

Equation 1 incorrectly read:

$$
\mathrm{CMRO}_{2}(t)=\mathrm{CBF}(t) \times \frac{\operatorname{Hbt}(t)}{\operatorname{Hbt}(t)} .
$$

The correct form of Eq. (1) is:

$$
\mathrm{CMRO}_{2}(t)=\mathrm{CBF}(t) \times \frac{\operatorname{Hbr}(t)}{\operatorname{Hbt}(t)}
$$

This has been corrected in the PDF and HTML versions of the Article.

Published online: 19 July 2021

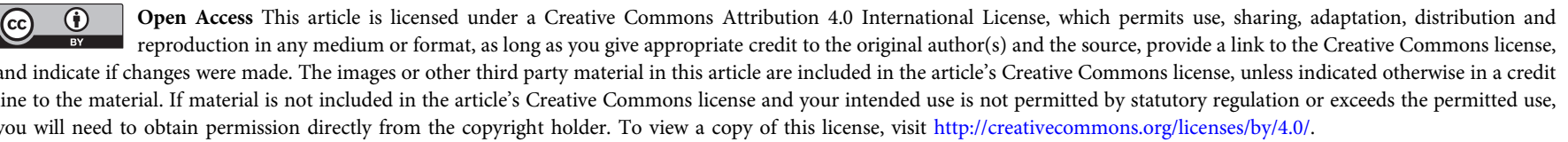

(c) The Author(s) 2021 